\title{
Genetics of epigenome might hold the clue for determining the threshold of environmental impact
}

\author{
Moinak Banerjee*,1 \\ ${ }^{1}$ Human Molecular Genetics Laboratory, Rajiv Gandhi Center for Biotechnology, Thiruvananthapuram, India \\ *Author for correspondence: Tel.: +91 471252 9529; mbanerjee@rgcb.res.in
}

First draft submitted: 7 May 2019; Accepted for publication: 4 June 2019; Published online: 5 July 2019

To determine the epigenetic basis of any disease one needs to identify the epigenetic landscape of the environment that drives the pathology. The epigenetic landscape of a disease can be determined by evaluating the alteration in DNA methylation, histone modifications and noncoding RNA expression. These molecular parameters of epigenomics have been monitored in response to the environment as a whole, while the role of genetics that govern these epigenomic parameters have often been ignored. We know that schizophrenia can be caused by a variety of environmental risk factors starting at early life complications and adding up with later life stressors. The early life events include prenatal infections, fetal hypoxia, prenatal maternal nutrition, maternal life stressors, birth season and location, while late life events include psychological stress, personality traits and substance abuse. There is no one to one correlation of these environmental risk factors in relation to epigenetic landscape. When schizophrenia arises, this could be due to the influence of many of these early and late life events and the epigenetic landscape might be the signature or combination of some of the risk factors. Therefore, monitoring each environmental factor or their combinations (risk or nonrisk) over a continuous time interval, and then relating them to shifts and balances in epigenetic landscape can be very challenging, as these factors are very dynamic and will also impact the epigenome dynamically [1]. The dynamics of environmental factors is difficult to control but how the host responds to these environmental factors might be relatively simpler as they are governed by the genetic factors that govern the epigenome. Today, we have precise understanding of the molecular indicators of the epigenome but correlating these molecular indicators to a specific environmental risk factor seems to be an enigma. Due to lack of this knowledge on the environmental correlates and its epigenetic indicators one often finds contrasting observations on the patterns of DNA methylation, histone modifications and noncoding RNA expression for any given complex disease like schizophrenia [2]. It is known that DNA sequence variations, epigenetic regulations and environmental cues act stochastically to contribute in the etiopathogenesis of schizophrenia [3]. Therefore, one of the simplest methods of epigenetic stratification would be to identify the genetic correlates of these epigenetic modifiers and modulators and stratify the study population genetically. This important aspect relating the genetic correlates of epigenetic modifiers and modulators has often been ignored when addressing the epigenetic indicators of health and disease.

We know that the epigenetic machinery is under the genetic control of classes of genes that are referred as writers, readers, erasers and remodelers. DNA methylation is controlled intrinsically by DNA methyltransferases, such as DNMT1, DNMT3A and DNMT3B that are involved in writing the DNA cytosine methylation on CpG dinucleotides and read by proteins that contain methyl-binding domains such as MECP2 and erased by the TenEleven Translocation enzymes through the formation of intermediates (5-hydroxymethylcytosine, 5-formylcytosine and 5-carboxylcytosine). Emerging evidences do indicate the role of genetic variation in influencing methylation level differences [4]. In schizophrenia and autism, altered methylation states have been reported and recent studies show that DNMT allelic variants with altered functions are associated with schizophrenia [5] and autism [6]. These genetic variants also have a transgenerational impact on methylation. Maternal genotypes of DNMT1 rs2241531 and DNMT3B rs6058897 were reported to influence the fetal global DNA methylation levels [7]. Even in a longitudinal study spanning 16 years, shows familial clustering in the degree of change in DNA methylation levels over time [8]. Similarly, among the reader cluster of genes such as MECP2, more than 500 disease-causing missense variants, deletions and duplications have been reported [9]. Some studies are also emerging with reference to erasers 
of methylation. A recent study indicates that TET2 rs2454206 genotype varied by ethnicity with AA genotype being higher in black individuals (79\%) and lesser in white individuals (39\%). This SNP is also known to play a role in epigenetic regulation of myelopoiesis [10].

DNA methylation is also extrinsically maintained by the folate pathway genes. SNPs in folate metabolizing enzymes like MTHFR, MTR and MTRR are reported to be associated with increased risk of psychiatric disorders. The influence of MTHFR on psychiatric diseases is mainly by way of reduction of MTHFR activity which results in elevation of homocysteine, reduction of DNA methylation-dependent methyl donor and finally by inducing hypomethylation, and activating disease-related genes [11]. MTHFR gene polymorphism has been linked to onset, clinical symptoms, prevalence as well as a response to treatments in schizophrenia [12]. The variants in folate pathway genes MTHFR 677T, MTR 2756A, FOLH1 484C, COMT 675A were found to be predictors of symptom severity in patients with low folate levels [13]. Even the SNPs in the CpG sites are important while evaluating environmental impact on methylome. CpG-SNP rs3796293 showed a pattern where methylation increased with the number of CpGs [14]. Therefore, if one tries to understand the impact of global or gene-specific methylation for any disease or environment, it would be ideal to understand it in the background of genetic variants of these drivers of DNA methylation, and subsequently develop a strategy to resolve the role of these genetic variants in defining the host response threshold to an environmental challenge. This would minimize the complexities of host methylation response to a large extent.

The histone machinery comprises writers, erasers, readers and remodelers. Writers include histone acetyltransferases and histone methyltransferases while erasers include histone deacetylases and histone demethylases. The readers are recognized as domains such as chromo domain, Tudor domain, MBT domain or PhD domain that have a high affinity for sites of histone methylation or Bromo domains that have high affinity for sites of acetylation. While the remodelers include ATP-dependent chromatin remodelers that play a crucial role in nucleosome maintenance. There are 73 histone acetylation modulator protein (HAMP) genes, that include 18 HAT genes, 43 BRD-containing genes (including 6 HATs that also contain BRDs) and 18 HDAC genes. Among these, HAMP proteins 63 have been involved in recurrent alterations in at least 1 cancer type and 16 HAMPs have been identified as putative therapeutic targets across multiple cancer types [15]. The majority of the studies on HAMP proteins have been restricted to cancer, but these genes are also known to regulate the expression of genes associated with brain function. Several lines of evidence on the role of histone modifications linked to candidate genes of schizophrenia have recently been discussed [2]. Elevated levels of HDAC1 are reported in the prefrontal cortex of SCZ patients. Also, the mRNA expression level of GAD67 was negatively correlated with the mRNA expression levels of HDAC1, HDAC3 and HDAC4 levels [16]. SNPs in these genes can influence the HDAC expression or the regulation of gene expression patterns relevant for brain function. SNPs in the HDAC3 and HDAC4 genes are reported to play a role in the pathophysiology of schizophrenia in a Korean population [17]. HDAC9 gene deletion has been reported to be associated with a small subset of schizophrenic patients [18]. A rare missense variants in the euchromatic histone lysine methyltransferases such as EHMT1 and EHMT2 genes were found to be associated with ASD patients [19]. Interestingly, these genes have been reported to have altered expression in schizophrenia with increased expression of EHMT2 in lymphocytes and the EHMT1 in both lymphocytes and postmortem parietal cortex [20]. A recent study suggested that among the three types of HAMPs; BRD proteins and HATs had the highest frequencies of recurrent mutations while HDACs had few recurrent events [15]. Polymorphisms in the transcription factor binding sites have also been reported to correlate with differences in local histone modification [21]. Very limited study exists with reference to genetic variants in histone modifier genes and psychiatric or neurodevelopmental disorders. Therefore, relating the role of genetic variants of histone modifier genes might be the critical first step to evaluate the influence of environment in driving these pathologies.

MicroRNA expressions have also been found to be altered in schizophrenia. Some of the drawback of these miRNA studies have been discussed as miRNA expression dynamically shifts its pattern with the corresponding environment [2]. miRNA expressions are also heavily modulated by miRNA target site mutations. A substantial number of SNPs and rare mutations within pri-, pre- and mature miRNA sequences and their relation to human diseases has been reported [22]. These SNPs can create new miRNA target sites, cause loss of miRNA target sites or effect miRNA:mRNA interaction. Some of these SNPs in miRNA target sites of host gene sequences can confer an increased risk of developing schizophrenia which can influence expression of small nucleolar RNA and long noncoding RNA [23]. Deletion of 22q11.2 has been reported to result in alterations in brain miRNA biogenesis and downregulation of miR-185 in SCZ-associated brain regions [24]. Controlling the environment and its impact 
on miRNA expression will be a challenging task but relating the target site mutation to miRNA expression might help resolve the interindividual variation in miRNA expression and its corresponding gene expression.

Considering these observations on the role of genetic variants in genes involved in DNA methylation, histone modifications and target site variations in miRNA-binding sites, it is highly appropriate to first investigate the role of these variants in disease pathogenesis as these variants would be the primary determinants of the epigenetic threshold. Once these thresholds are surpassed only then the impact of environment can be considered to play a role in pathogenesis. Interpretation of DNA methylation, histone modifications and miRNA expression should be first subjected to their genetic background. Understanding this aspect of genetic control of methylation, histone modifications and miRNA expression or for that matter epigenetics might help in defining the individual's threshold to withstand the environmental stress. In addition, it might also help in defining the limits of genetic control of the epigenome and identify the environmental load for any given disease.

\section{Acknowledgments}

Due to limitation of space we could not cite several authors whose work also contributes to similar observation.

\section{Financial \& competing interests disclosure}

The authors have no relevant affiliations or financial involvement with any organization or entity with a financial interest in or financial conflict with the subject matter or materials discussed in the manuscript. This includes employment, consultancies, honoraria, stock ownership or options, expert testimony, grants or patents received or pending, or royalties.

No writing assistance was utilized in the production of this manuscript.

\section{References}

1. Cortessis VK, Thomas DC, Levine et al. Environmental epigenetics: prospects for studying epigenetic mediation of exposure-response relationships. Hum. Genet. 131, 1565-1589 (2012).

2. Swathy B, Banerjee M. Understanding epigenetics of schizophrenia in the backdrop of its antipsychotic drug therapy. Epigenomics 9 (5), 721-736 (2017).

3. Petronis A. The origin of schizophrenia: genetic thesis, epigenetic antithesis, and resolving synthesis. Biol. Psychiatry 55, 965-970 (2004).

4. Coolen MW, Statham AL, Qu W et al. Impact of the genome on the epigenome is manifested in DNA methylation patterns of imprinted regions in monozygotic and dizygotic twins. PLoS ONE 6, e25590 (2011).

5. Saradalekshmi KR, Neetha NV, Sathyan S, Nair IV, Nair CM, Banerjee M. DNA methyl transferase (DNMT) gene polymorphisms could be a primary event in epigenetic susceptibility to schizophrenia. PLoS ONE 9(5), e98182, (2014).

6. Alex AM, Saradalekshmi KR, Shilen N, Suresh PA, Banerjee M. Genetic association of DNMT variants can play a critical role in defining the methylation patterns in autism. IUBMB Life 10.1002/iub.2021 (2019) (Epub ahead of print).

7. Potter C, McKay J, Groom A et al. Influence of DNMT genotype on global and site specific DNA methylation patterns in neonates and pregnant women. PLoS ONE 8(10), e76506 (2013).

8. Bjornsson HT, Sigurdsson MI, Fallin MD et al. Intra-individual change over time in DNA methylation with familial clustering. JAMA 299, 2877-2883 (2008).

9. Sawalha AH, Webb R, Han S et al. Common variants within MECP2 confer risk of systemic lupus erythematosus. PLoS ONE 3(3), e1727(2008).

10. Kutny MA, Alonzo TA, Gamazon ER et al. Ethnic variation of TET2 SNP rs2454206 and association with clinical outcome in childhood AML: a report from the Children’s Oncology Group. Leukemia 29(12), 2424-2426 (2015).

11. Wan L, Li Y, Zhang Z, Sun Z, He Y, Li R. Methylenetetrahydrofolate reductase and psychiatric diseases. Transl. Psychiatry 8(1), 242(2018).

12. Klengel T, Pape J, Binder EB, Mehta D. The role of DNA methylation in stress-related psychiatric disorders. Neuropharmacology 80, 115-132 (2014).

13. Roffman JL, Brohawn DG, Nitenson AZ, Macklin EA, Smoller JW, Goff DC. Genetic variation throughout the folate metabolic pathway influences negative symptom severity in schizophrenia. Schizophr. Bull. 39(2), 330-338 (2013).

14. van den Oord EJ, Clark SL, Xie LY et al. A whole methylome CpG-SNP association study of psychosis in blood and brain tissue. Schizophr Bull. 42(4), 1018-1026 (2016).

15. Hu Z, Zhou J, Jiang J et al. Genomic characterization of genes encoding histone acetylation modulator proteins identifies therapeutic targets for cancer treatment. Nat Commun. 10(1), 733 (2019)

16. Sharma RP, Grayson DR, Gavin DP. Histone deactylase 1 expression is increased in the prefrontal cortex of schizophrenia subjects: analysis of the National Brain Databank microarray collection. Schizophr. Res. 98(1), 111-117 (2008). 
17. Kim T, Park JK, Kim HJ, Chung JH, Kim JW. Association of histone deacetylase genes with schizophrenia in Korean population. Psychiatry Res. 178(2), 266-269 (2010).

18. Lang B, Alrahbeni TMA, Clair DS et al. HDAC9 is implicated in schizophrenia and expressed specifically in post-mitotic neurons but not in adult neural stem cells. Am. J. Stem Cells 1(1), 31 (2012).

19. Balan S, Iwayama Y, Maekawa M et al. Exon resequencing of H3K9 methyltransferase complex genes, EHMT1, EHTM2 and WIZ, in Japanese autism subjects. Mol. Autism 5(1), 49 (2014).

20. Chase KA, Gavin DP, Guidotti A, Sharma RP. Histone methylation at H3K9:evidence for a restrictive epigenome in schizophrenia. Schizophrenia Res. 149(1-3), 15-20 (2013).

21. McVicker G, van de Geijn B, Degner JF et al. Identification of genetic variants that affect histone modifications in human cells. Science 342(6159), 747-749, (2013).

22. Moszyńska A, Gebert M, Collawn JF, Bartoszewski R. SNPs in microRNA target sites and their potential role in human disease. Open Biol. 7(4), 170019 (2017).

23. Gibbons A, Udawela M, Dean B. Non-coding RNA as novel players in the pathophysiology of schizophrenia. Noncoding RNA 4(2), 11 (2018).

24. Forstner AJ, Degenhardt F, Schratt G, Nöthen MM. MicroRNAs as the cause of schizophrenia in 22q11.2 deletion carriers, and possible implications for idiopathic disease: a mini-review. Front. Mol. Neurosci. 6, 47 (2013). 